\title{
22
}

\section{Current Technologies for On-Line Monitoring of Drinking Water in Distribution Systems}

\author{
Mirnader Ghazali and Edward A. McBean
}

Increased risks of drinking water contamination are incentives for implementation of improved monitoring approaches for pathogens in drinking water distribution systems. However, indirect measurements such as $\mathrm{pH}$, and turbidity to detect pathogens do not guarantee the safety of drinking water because pathogens, and especially protozoa and viruses, are not well correlated to these indirect measures. Routine methods for culturing indicator micro-organisms need considerable time and hence do not provide significant value for real-time monitoring efforts. Review of currently available technologies (spectral fluorescence laser technology, image processing technology, multi-angle light scattering laser technology, on-line chemical characterization system, decision support monitoring system, and rapid response tests for total micro-organisms and toxicity) for real-time and/or rapid pathogen detection, demonstrates there is no single instrument currently available. The most effective approach currently available is demonstrated to involve the identification of the presence of a pathogen, and the instigation of collection of a sample for more detailed analyses for confirmation.

Ghazali, M. and E. McBean. 2009. "Current Technologies For On-Line Monitoring of Drinking Water Distribution Systems." Journal of Water Management Modeling R235-22. doi: 10.14796/JWMM.R235-22. (C) CHI 2009 www.chijournal.org ISSN: 2292-6062 (Formerly in Conceptual Modeling of Urban Water Systems. ISBN: 978-0-9808853-2-3) 


\subsection{Introduction}

There is increasing interest in microbiological and/or chemical contamination detection capability in drinking water systems. Typically, municipalities use $\mathrm{pH}$, turbidity, and free or total chlorine to indicate water quality at the source and rely on chlorination to maintain this quality in the distribution system. However, newly-identified pathogens and the risk of contamination by bioterrorists or disgruntled employees necessitate more attention to drinking water quality and better monitoring approaches, especially for pathogens (Payment, 1999).

The degree of public confidence in drinking water supplies is substantial, but if this confidence is lost as the result of an incident of contamination, it may be extremely difficult to regain. Events in Walkerton (May 2000) and North Battleford (April 2001) are two of a number of recent examples of microbial contamination of drinking water. In Walkerton, seven people died and more than 2,300 people became ill after drinking water contaminated by Escherichia coli O157:H7. An investigation revealed that the outbreak could have been prevented if free chlorine in the water supply was monitored daily. In the second case, that for North Battleford, more than 7,000 people became ill due to the contamination of drinking water by Cryptosporidium parvum (Krewski, et al. 2004). Numerous other events have been reported (e.g. in Schuster et al. (2005)) and it is clear that real-time monitoring or rapid detection systems in drinking water distribution systems would be invaluable for prevention of future water-related illnesses in Canada. Implementation of such systems is recommended as the first step in the development of Canadian drinking water protection (Krewski, et al. 2004).

Chemical measurement technologies have real-time capability but the availability of such technologies for pathogen detection is limited. Some microbial measurements (e.g. E.coli) can be used to indicate the presence of other bacterial pathogens, but are less effective at predicting risk from protozoa and viruses (Payment, 1999). Further, in any type of monitoring system that involves, a delay between the time of sample collection and the time when the results are obtained, contaminated water may be distributed to the consumers before there is knowledge of any danger (Krewski, et al. 2004). This is an especially important issue in pathogen detection, where many of the current detection/identification procedures require days for identification of an issue. Due to the need for rapid tests, and real-time monitoring of drinking water at the source and in distribution systems, there is significant ongoing research into the development of appropriate instrumentation. This chapter reviews currently available technologies and 
some soon-to-be-released technologies aimed at providing real-time and/or rapid quantification of microbial pathogens.

\subsection{Background}

The purposes of monitoring water quality in water distribution systems include providing early detection of a problem, and reducing or eliminating the risk of outbreaks. Monitoring the water at the source can lead to effective protection by using those results to ensure that appropriate treatment processes are in place, and monitoring of water quality within the distribution system itself will allow assessment of system integrity and identification of areas facing water quality deterioration (Krewski, et al. 2004, Huang and McBean 2007).

The causes of a number of outbreaks in the U.S. and throughout the world have been determined, in the hope of decreasing the risk of future outbreaks by employing proper actions. Hrudey and Hrudey (2007) reviewed 73 documented outbreaks from the past 30 years to determine the major contributing factors. In all cases, more than one factor was involved. Some of the factors included: cross-connections and distribution failures, wastewater contamination, inadequate disinfection, extreme weather, inadequate knowledge of source water hazards, filtration failures, wildlife or livestock fecal contamination, and changes in plant maintenance or treatment processes. In another study, Craun et al. (2002) reviewed outbreaks in drinking water systems in the U.S. between 1991 and 1998, and concluded that outbreaks were most often caused by failure in the treatment system or inadequate treatment of surface or groundwater, contamination of water in the storage tanks, and cross-connections and corrosion within the distribution system. Several studies have suggested that most of the outbreaks were caused by similar factors that were not monitored or not identified in time. It was concluded that most of these outbreaks could have been prevented by more effective monitoring of contaminants in the water within distribution systems.

The need for real-time monitoring of drinking water quality in distribution systems is significant, considering Canada's deteriorating infrastructure. Low investments in maintenance and repair of public water infrastructure during the past decades have now created a need for significant investment to maintain the public water infrastructure. For example, in the central portions of many major cities, many pipes are 
more than 100 years old (McBean 2006). According to the U.S. EPA, the required investment in the U.S. alone is at least $\$ 151$ billion over the next two decades. The need for maintenance of wastewater infrastructure is even larger due to the high risk of cross-connection, in addition to the necessary replacement of aging facilities (Levin, et al. 2002). Therefore, as the water mains and pipes in the distribution system are getting older, the rate of deterioration as well as the risk of breakage is increasing; however, precise prediction needs comprehensive data (Kleiner and Rajani 2001; McBean 2006).

Contamination of surface and ground water supplies is another important issue that increases the need for real-time monitoring of the water. The contamination may be the result of hazardous waste sites, industrial activities, transportation and residential developments; agricultural activities; or animal husbandry. Contamination may also result from naturally occurring trace metals, such as arsenic (Levin, et al. 2002). Intentional contamination of drinking water by bioterrorists or disgruntled employees is also of increasing concern (McBean 2006).

All of the aforementioned risks demonstrate the importance of real-time monitoring of drinking water quality. An ideal water quality monitoring system would be an integrated system including measuring devices and/or sensors for the detection of pathogenic bacteria, viruses, and protozoa, as well as toxins and potentially harmful chemicals. In recent years, extensive research to develop instruments for the rapid detection of these contaminants has been ongoing. As a result, some technologies for rapid or real-time detection of chemicals and pathogens have been developed, and are critically assessed below.

\subsection{Available Instruments and Analytical Methods}

No single method or instrument is able to detect all the contaminants in water. Even categorizing contaminants into chemical and biological agents does not solve the problem, which means the monitoring of water for all, or most, contaminants requires a series of complex instruments that are both expensive and tedious (Lemarchand et al. 2004). For this reason, reliance is often placed on 'indirect' indicators to detect changes in the quality of the water in real or near real-time (Skadsen et al., 2008). However, there are some challenges and questions in real-time monitoring of these "indirect" indicators (Straub and Chandler, 2003) as follows: (i) what are the detection objectives? e.g. enumeration of the biological target or measuring the 
concentration of a chemical contaminant; determining the viability and infectability of target microorganism or presence/absence test; (ii) what is the acceptable level of risk in detection? e.g. the acceptable level of false positive/false negative rate; and (iii) how the sample should be taken in order to represent the real situation.

There has been considerable research on the development of systems with the capability to detect or measure the contamination levels in drinking water. These include, for example, lab-on-a-chip devices for microbial monitoring and detection in water (Liu and Lay, 2007); quartz crystal microbalance biosensor for bacterial detection (Shen et al., 2007); PCR techniques (Guy et al., 2003); and oligonucleotide based microarrays (Maynard et al., 2005). Most of these systems are in the research or development stages and are expected to be introduced for public use in the near future. In this chapter, we explain the systems which are commercially available and can be used alone, or in combination with other technologies to monitor the drinking water in real or near real-time. Some of the most important available technologies for real-time monitoring approaches include the following.

\subsubsection{Spectral Fluorescence Laser Technology}

Laser induced fluorescence technology has been used for several purposes in the field of water monitoring including, for example, the measurement of excitation emission matrix spectra of dissolved organic carbons in water (Killinger and Sivaprakasam, 2006). The signal obtained from the sample, spectral fluorescence signatures (SFS), has been used for substance recognition, and the level of the fluorescence intensity has been used for quantity measurement. A sample's SFS is measured and stored in an electronic spectral library. Software compares a sample's fluorescence pattern to the library of known compounds in order to identify the substance. The calibration curve, created for that material using standard samples, is used to quantify the target in the sample.

With this procedure, sample preparation is not needed, and the analysis requires about five minutes in elapsed time. Sampling can be automated using an automated sampler and a flow-through system. Therefore, it can be used for near real-time monitoring. Detection sensitivity is in the range of one part per million ( $\mathrm{ppm}$ ) to one part per billion ( $\mathrm{ppb}$ ) for aromatic organic chemicals (Killinger and Sivaprakasam, 2006), but its application in the detection and quantification of biological material is not yet fully established. One of the issues is that for pathogen detection, the 
concentration of microorganisms must be high, a significant issue unless the concentrations of the pathogens are initially very high.

In order to determine the applicability of this instrument for the detection and quantification of biological material, samples containing E.coli (ATCC 11775) were tested using the Instant Screener system (Laser Diagnostic Instruments International Ltd.). For these tests, E.coli was cultured using nutrient broth (Difco ${ }^{\mathrm{TM}}$ ) at $32{ }^{\circ} \mathrm{C}$ for 24 hours and three different concentrations, low, high, and very high, were prepared using pure sterilized water from fully grown culture. Since the sensitivity of the instrument for microorganisms was not known, preliminary random tests were carried out in order to determine the minimum concentration of E.coli to get the SFS. The lowest concentration was determined to be at least more than $10^{6} \mathrm{CFU} / \mathrm{ml}$. The results are shown in Figure 22.1 containing two dimensional spectra for the three samples. The $\mathrm{Y}$ and $\mathrm{X}$ axes indicate excitation and emission wavelengths respectively. Laser induced excitation and emission were in the range of $240-360 \mathrm{~nm}$ and $265-$ 585 , respectively, with a spectral resolution of $3.5 \mathrm{~nm}$ and response time of $5 \mathrm{~min}$. The circled areas show the special points related to the E.coli SFSs. These points are stored and can be used for the calibration and detection of unknown samples. Although the SFS results show different intensities for different concentrations of E.coli, higher precision, e.g. $1 \mathrm{~nm}$ resolution, and sample concentration would be required in order to use this system in an on-line monitoring system. This is because the concentration of microorganisms in the drinking water may be lower than the detection limit of the instrument on the one hand and, on the other hand, real samples contain other compounds which may interfere with the SFS for the target microorganism. There is ongoing research to determine the prediction power of this system in the detection and quantification of a biological sample in tap water as well as the interaction of SFSs when there are simultaneously, both chemical and biological contamination.

Further complicating the detection of pathogens is the fact that a calibration curve for each substance is needed, and the library for target substances still needs to be created. The price of this equipment is in the range of $C \$ 30,000-50,000$ and using an instrument with higher resolution increases the cost. For example, an instrument with $1 \mathrm{~nm}$ resolution of the spectrum will cost around $\mathrm{C} \$ 100,000$. The higher resolution is needed when there are more target microorganisms to be monitored in order to differentiate their SFSs within a small range. 


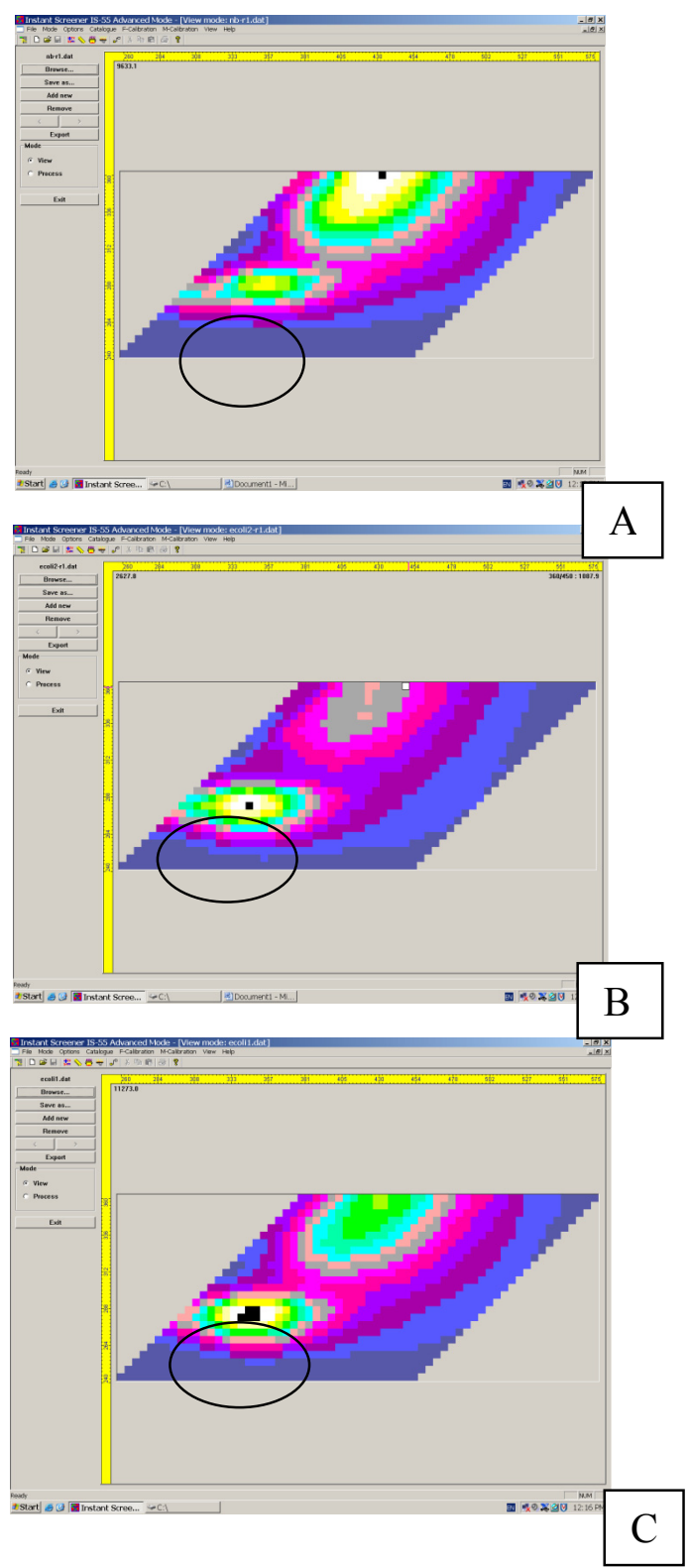

Figure 22.1 Spectral Fluorescence Signature (SFS) for three different concentrations of E.coli: A: low, B: high and C: very high. 


\subsubsection{Image Processing Technology}

Image processing has received significant attention as an analytical and monitoring tool (Pilloti et al., 1997). In water quality monitoring, image processing is being used to capture images as the water passes through a flow-through cell (with a depth of 100 or $400 \mu \mathrm{m}$ ), allowing automatic analysis of suspended particulates in a liquid sample. A digital microscopy system is used to capture images of the particles suspended in the fluid sample using a 10bit grayscale camera. The images are then analyzed to create a database consisting of particle count, size, and morphological information such as transparency, perimeter, area, and circularity (in terms of how close the particle is to a circle). Figures 22.2 and 22.3 show the results obtained for a sample containing high concentrations of E.coli in pure water. In Figure 22.2, the number of particles in each image is shown in different size categories (bins), and Figure 22.3 shows the cumulative number of particles in each category (bin). Due to the limitation of the instrument in categorizing the particles with sizes less than $1 \mu \mathrm{m}$, all the particles up to $1 \mu \mathrm{m}$ are accumulated and reported in the first bin with a size distribution of $1-2.25 \mu \mathrm{m}$. This is one of the limitations of this equipment for monitoring microorganisms, especially for those with the size of smaller than $1 \mu \mathrm{m}$.

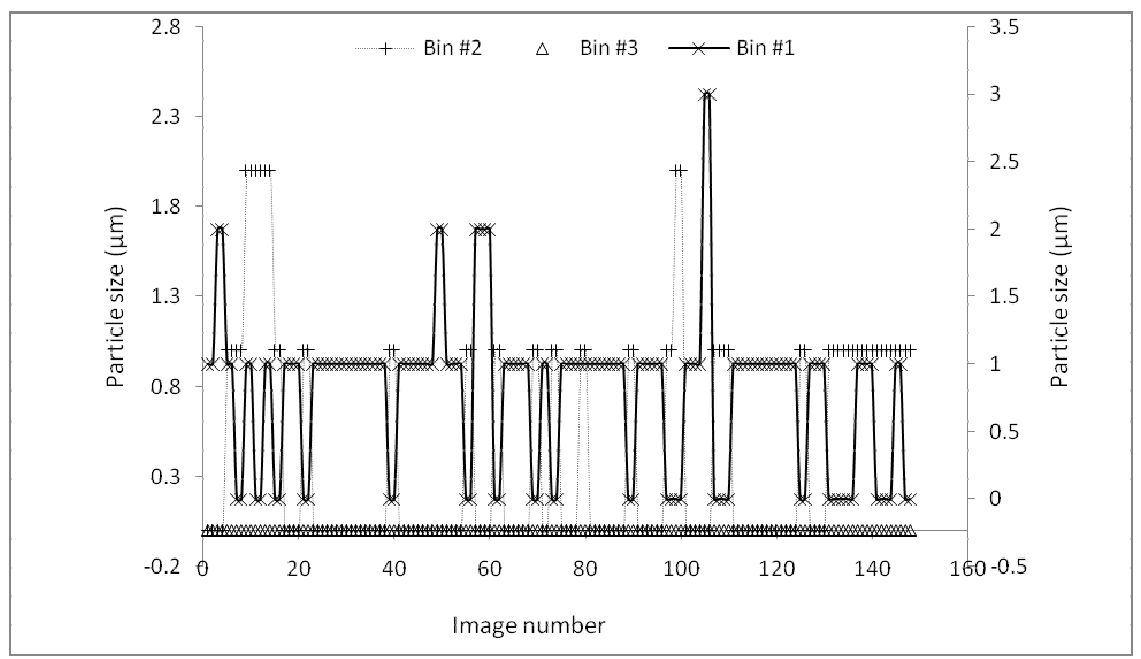

Figure 22.2 Particle size analysis for a sample containing high concentration of E.coli. Bin \# 1: 1-2.25 $\mu \mathrm{m}$, Bin \# 2: 2.25-3.25 $\mu \mathrm{m}$, and Bin \# 3: 3.25-4.25 $\mu \mathrm{m}$. 


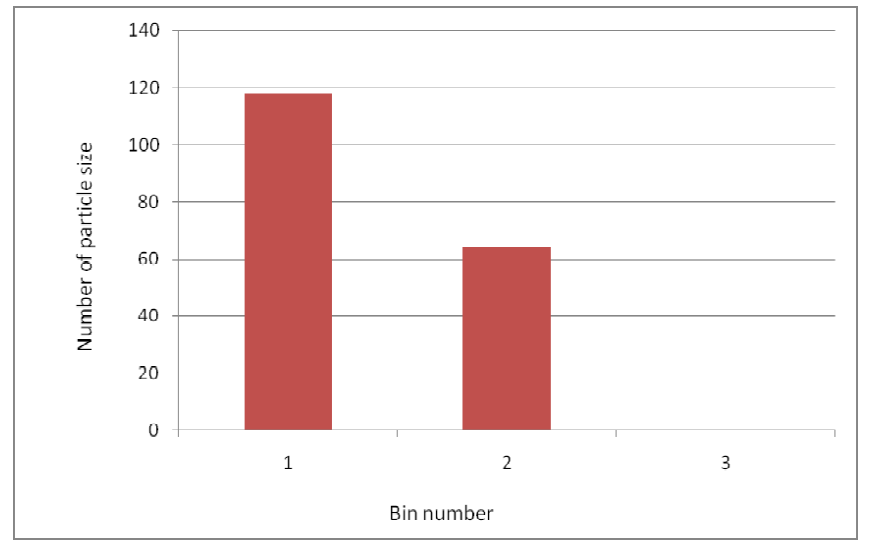

Figure 22.3 Cumulative particle size analysis for the sample with high concentration of E.coli in three categories (bins) including Bin \# 1:

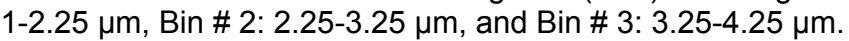

Software analyzes the data and produces different types of information such as size distribution trend charts, morphological parameter histograms and scatter plots, and the real-time display of suspended particles as shown in Figures 22.2 and 22.3. Digital filtering can be used for particle characterization by isolating sub-populations of interest, e.g. different ranges of sizes, shapes etc. Calibration is not needed and particle concentrations are determined from the images.

Different magnifications can be used for different particle-size ranges from 1 to $400 \mu \mathrm{m}$. This particle-size range covers most protozoa, but not all bacteria, and viruses. The rate of analysis (by volume of water) varies, decreasing with increasing magnification, and is in the range of 5 to $200 \mu \mathrm{l} / \mathrm{min}$. Hence this system is most suitable for the monitoring of fluid samples to determine the concentration of suspended particles and to obtain general information about the morphology of the particles. One of the limitations of this instrument is the reliance upon a 10bit grayscale camera that does not have the capability of color imaging. Color images would give improved results where an indicator color would be needed to differentiate the microorganisms or to detect the live cells from other particles. This improvement would require increased memory size, more advanced technology to capture and analyze high resolution color images, and possibly on-line coloring of live microorganisms using a suitable color, which would increase the price of the system. Limitations also exist in the fact that there is only one camera and the images are a 2-D representation of a 3-D volume of 
fluid; therefore, particles are only observed from one perspective, making it difficult to differentiate microorganisms from particles. Also, the system cannot be plumbed directly into a closed circuit system. Automated sampling is possible by adding an automatic sampler with which the sampling can be programmed and samples can be grabbed automatically e.g. by using programmable peristaltic pumps. Limitations of this procedure include:

(1) Currently, continuous imaging of the sample passing through the flow cell is possible for a maximum of just 24 hours due to limits in memory size. Further improvements in imaging technology and storage capacity of the equipment are needed for potential use of this instrument in real-time monitoring of drinking water for lengthy periods of time.

(2) This instrument monitors only particles, which means it cannot be used for dissolved contaminants such as chemicals. Therefore, additional instruments for the detection of toxins are needed to monitor water quality. The price of the current system is in the range of $C \$ 50,000$ to 70,000 .

\subsubsection{Multi-angle Light Scattering Lasers}

Multi-angle light scattering (MALS) technology is another technology which has been developed for continuous, and real-time monitoring of water. This system uses laser-produced MALS technology for pathogen detection and classification (not identification). MALS technology is based on laser technology, particle light scattering, photo-detection and computer signal processing. Unlike the spectral fluorescence laser technology which uses excitation emissions of compounds for the identification and quantification of substances, MALS technology uses the light scattering pattern of a substance for detection and identification. This device uses a laser beam which is focused into a slip-stream of water. When a small particle such as a microorganism passes through a laser beam, it generates a unique pattern called a Bio-Optical Signature (BOS). This signature is unique for different classifications of micro-organisms. A pathogen detection library is used for the classification of the micro-organism. This system needs to be calibrated for any specific site location where the technology might be applied because of different background particle counts 
or noise. The lowest concentration of E.coli detectable by this system is $600 / \mathrm{ml}$ which means the sample needs to be concentrated (Adams and McCarty, 2007).

This instrument may also be integrated into information systems for communication, alerting or notification purposes. In fact, one purpose of this system is to act as an 'alarm box' to warn of a probable pathogen 'event'. In the case of an event, the system can capture a water sample for more detailed analysis. No reagents are needed and the equipment needs only routine cleaning, the frequency of which depends on the water quality. Information is refreshed every minute and shows microbial counts for the species being monitored as well as unclassified microorganisms. This device is designed to work for water with background particle counts normally of less than $1,000 / \mathrm{ml}$.

The current pathogen library includes protozoa (Cryptosporidium parvum, and Giardia), and bacteria (E.coli, Salmonella, Shigella, Pseudomonas and Legionella). It should be noted that the accuracy of this system is not equal to the accuracy of laboratory methods, which means there is a need for validation. This technology has been developed primarily for contamination warning; therefore, this equipment is not able to detect pathogens at the current time at levels of concern. However, the instrument can be used for real-time warning, to identify an event which might go unnoticed for a while if there was no such system. Therefore, in the case of an 'alert condition', confirmation tests are recommended. This system works only for micro-organisms and particles, and is not effective at detecting chemicals. The detection time and the probability of classifying a particle is proportional to the concentration of the microorganism which means low concentrations will take a much longer time to detect. For example, with current technology the detection time for the concentrations of 1, 4 and 40 particles/L will be $4 \mathrm{~h}, 1 \mathrm{~h}$, and 5 minutes, respectively. The price of a commercial system ranges from $C \$ 35,000$ to 50,000 , depending on the options and specifications.

\subsubsection{On-Line Chemical Characterization System}

Some commercial systems have used routine off-line measurements in online mode and for real-time monitoring of drinking water distribution system (Skansen et al., 2008). These tests have been automated and integrated into a package that can be used as an early warning system. In one available system, five common parameters including chlorine (free or total), $\mathrm{pH}$, turbidity, conductivity, and total organic carbon (TOC) are measured and a 
baseline for water quality is created at normal operating conditions. Then, every 60 seconds, it analyzes sensor data calculating deviations from a water quality baseline. One of the important factors in designing such a system is dealing with background variability which should be determined before operating the system in real condition. As well, it is desirable to use an appropriate algorithm such as Baysian Belief Network to determine the false positives which are a threat for any on-line real-time monitoring system (Pike, 2004). The system analyzes the agent library and plant library to assess and classify the deviation to determine the merit in creating an alarm. The agent library includes fingerprints for different contaminants mostly toxins and herbicides. If the software doesn't find any match in the agent library, it stores the deviation in its library for future reference. This system requires little maintenance, but is not capable of pathogen detection or classification and quantification. Therefore, there needs to be secondary instrumentation for confirmation of the contamination by pathogens.

\subsubsection{Decision-Support Monitoring System}

Another commercial system uses a similar approach as described in the previous section but with different data inputs. As an example, Bidewell (2000) has described a decision support system for protecting groundwater from land treatment of wastewater. This system uses software that receives different inputs such as sensor data, lab results, information about the site, surrounding environment, treatment system, distribution system and water usage. This software can be called a data monitoring system. The inputs are used to interpret the results obtained from sensors or lab results. The setup for this system requires a comprehensive site audit, before any installation to establish normal operating conditions. The sensing parts are designed to capture relative information, giving more weight to data analysis and correlation, than to signal analysis. In fact, this system is a decision support tool rather than an on-line monitoring system and the main purpose is the monitoring of data from various inputs to assess trends and identify events that are "out of pattern". In some of the systems, the sensing part contains LEDs and laser diodes, used for excitation, and silicone photodiodes. The data (signals) acquired from these sensors pass through digital signal processing spectroscopy (DSPS) circuits in order to translate the signals into interpretable results. For example, the result of yellow excitation is correlated to iron bacteria and humic material; infrared excitation is correlated to turbidity and suspended solids; UV excitation is correlated to nicotinamide adenine dinucleotide or $\mathrm{NAD}(\mathrm{H})$ which is a co-enzyme in 
some oxidation-reduction reactions; and blue LED on red filter has been used for chlorophyll and porphyrins. The results are integrated with a network to monitor the water quality continuously and report the data from a remote place using a wireless, dial up or Ethernet network. For the sensor data, the baseline could be the moving average of the results in comparison with a previous time period. In the situation of an event, confirmatory tests are necessary. Also, this system cannot directly detect pathogen contamination; therefore, either biosensors or confirmatory tests are needed for this purpose.

The security of data transferred through wireless network and over the internet may be compromised and so a backup plan needs to be considered. The price of a system may vary from less than $\$ 1,000$ to about $\$ 50,000$ for a high level system.

\subsubsection{Rapid Response Tests for Total Micro-organisms}

Another instrumentation type important in the monitoring of drinking water is rapid response tests or instruments. These tests do not monitor the water quality in real-time, but they can be used by an operator to rapidly determine the existence of a specific contaminant or to measure the general quality of water at a site. These systems are portable and results can be obtained and processed within minutes to a maximum of an hour. Of course, there are ongoing efforts to automate these types of monitoring systems; therefore, they can be used for real or near real-time on-line monitoring of drinking water.

A rapid response system has been developed to measure the total microorganism population in a water sample based on the measurement of adenosine triphosphate (ATP). ATP is a direct indicator of total biomass and is measured using the firefly luciferase assay. ATP is extracted and measured using the light produced from luciferin/luciferase enzymatic reaction (Delahaye et al., 2003). Each ATP molecule produces one photon of light and a light reader system measures the light. The cellular ATP is measured and correlated to the total biomass. For each set of analyses, a calibration is needed to calculate ATP from the Luminometer reading. In terms of microbial concentration, the sensitivity range is from 10 to $10^{9} \mathrm{CFU} / \mathrm{ml}$ (depending on the sample type) and the sensitivity can be increased by filtration if required. The sample volume required for a drinking water test is between 50 to $100 \mathrm{ml}$. This test kit is generally designed for determining the total microorganism content of the water in a short time, but in actuality, the measured value is total microbial energy 
rather than total microbial count since different microorganisms contain different amounts of ATP, primarily due to their different sizes, although this would not create a substantial error. This technology cannot detect a difference between pathogenic and non-pathogenic microorganisms. In comparison with other on-line systems, the price of this system is lower, at about $\mathrm{C} \$ 6,000$ which makes it affordable for small site monitoring. Test kits cost about C\$5-12 each.

\subsubsection{Toxicity Testing Using Luminescence}

Another portable and rapid response, but off-line system, has been developed for testing the toxicity of drinking water (Bulich and Isenberg, 1981). This system can rapidly test the toxicity of samples in about 30 minutes. In this system, luminescent bacterium (Vibrio fischeri) is used as an indicator. This bacterium produces light as a by-product of normal metabolic reactions. In the presence of toxins, normal metabolism will be inhibited and the light output will be decreased. Using a control reagent, the reduction in luminescence is measured using an instrument for reading the luminescence, and interpreted as the level of toxicity of the sample. Each measurement needs a reagent blank in order to compare the light output from the sample and a control. This system responds to a wide range of chemicals and toxicants such as pesticides, industrial chemicals, metals, fungicides and other similar materials. This system is restricted to testing for chemicals and toxins, not for pathogens and biological contamination. Ulitzur et al. (2002) have developed the same assay but using a more sensitive variant of luminescent bacterium, Photobacterium leiognathi, to detect very low levels of different toxins, less than a milligram per litre, in water.

These rapid response systems are useful when there is a need for such a measurement in less time, or for monitoring water quality for a specific period of time (such as conferences, athletic events etc.). Some of the limitations of these systems are: the reagent (for the toxicity test) must be kept in a freezer $\left(-20\right.$ to $\left.-25^{\circ} \mathrm{C}\right)$, and a reconstituted sample should be used within 30 minutes; turbid samples should be clarified and colored samples should be diluted which means they need longer assay time and other equipment as well; samples with chlorine should be dechlorinated using sodium thiosulfate solution; low, medium and high toxic samples need different procedures, making the measurement (toxicity test) difficult for unknown concentrations of chemicals or toxins in the samples, and may need repeating some of the tests to be repeated. 


\subsection{Examples of Scenarios}

While there is no single instrument to monitor all possible contaminants in the drinking water distribution network, this research has been identifying combinations of methods/instruments which can be used. However, the appropriate combination/procedure is site-specific and depends on factors including the level of protection, size of the distribution network, available funds etc. For example, for the highest possible level of protection, an online chemical characterization system along with suitable data processing can be used as the first step. Data processing is needed to detect the true and false positive responses. Also, a library of "fingerprints" can be used to categorize the contamination when there is a response indicating a change or "blip" in the water system. In the next step, an automatic sampler can be used to take the real time sample(s) when there is a "blip". Samples can be analyzed by different procedures/instruments based on the results obtained from the primary system and data processing unit. For example, spectral fluorescence laser technology can be mainly used for chemical contaminants and laser light scattering technology can be used to categorize the biological contamination. However, this combination requires a high investment and may not be applicable for regular systems while it can be useful for the monitoring of drinking water for special cases such as embassies, which may be of high interest for bioterrorists.

In a general case, the scenario may be defined as using a general chemical characterization system with signal processing to detect a "blip". Emergency responses can be managed based on the signal and at the same time some rapid response tests can be used for confirmation and determination of the level of contamination. In this case, where the drinking water distribution system is large, the number of the systems and their locations are critical to get the acceptable level of coverage and protection.

For some cases, only rapid response tests can be used with acceptable intervals to monitor the quality of water. For example a food factory producing a product which relies on the safety of the water can use the ATP or toxicity test twice a day to monitor the quality of water.

\subsection{Conclusion}

Of the technologies currently available or in development for the purposes of real-time and/or rapid pathogen detection, there is no single instrument or system that has the ability to monitor all the possible contaminants or threats 
in drinking water. Particular limitations exist with regard to the monitoring of pathogens. Consequently, it is appropriate to employ a series of technologies to monitor drinking water distribution systems. The most effective approach, given available technology, is to utilize real-time monitoring to identify the presence of a pathogen and, simultaneously, instigate collection of a sample for retrieval and more detailed analyses for confirmation. The capabilities of the various instruments to identify a 'blip' are definitely substantial, and can be reliably employed to indicate a problem in the quality of drinking water and the sounding of an alarm in near realtime as well as capturing a sample for subsequent rapid analysis and confirmatory tests.

\section{Acknowledgments}

Financial contributions from the NSERC Strategic Grants program are gratefully acknowledged.

\section{References}

Adams J. A. and McCarty D. 2007. Real-time, on-line monitoring of drinking water for waterborne pathogen contamination warning. Internat. J of High Speed Electronics and Systems., 17(4):643-659.

Bidewell V. J. 2000. Decision support for protecting groundwater from land treatment of wastewater. Environmetrics, 11:553-562.

Bulich A.A., Isenberg D.L. 1981. Use of the luminescent bacterial system for the rapid assessment of aquatic toxicity. ISA trans., 20(1):29-33.

Craun G. F., Nwachuku N., Calderon R. L., Craun M. F. 2002. Outbreaks in drinkingwater systems, 1991-1998. J Environ Health, 65:16-23.

Delahaye E. et al. 2003. An ATP-based method for monitoring the microbiological drinking water quality in a distribution network. Water research, 37:3689-3696.

Guy R. A. et al. 2003. Real-time PCR for quantification of Giardia and Cryptosporidium in environmental water samples and sewage. Applied and environmental Microbiology, 69(9):5178-5185.

Hrudey S. E., Hrudey E. J. 2007. Published case studies of waterborne disease outbreaksevidence of a recurrent threat. Water Environ Res, 79:233-245.

Huang, J. and E. McBean. 2007. "Water Quality Modeling using Fault Tree Method." Journal of Water Management Modeling R227-19. doi: 10.14796/JWMM.R227-19.

Kleiner Y., Rajani B. 2001. Comprehensive review of structural deterioration of water mains: statistical models. Urban Water, 3:131-150.

Killinger D. and Sivaprakasam V. 2006. How water glows: Water monitoring with laser fluorescence. Optics and Photonics News, 17(1):34-39. 
Krewski D, Balbus J, Butler-Jones D, Haas CN, Isaac-Renton J, Roberts KJ, Sinclair M. 2004. Managing the microbiological risks of drinking water. $J$ of Toxicology and Environmental Health, Part A, 67:1591-1617.

Lemarchand K., Masson L., Brousseau R.. 2004. Molecular biology and DNA microarray technology for microbial quality monitoring of water. Crit Rev Microbiol, 30:145-172.

Levin R. B., Epstein P. R., Ford T. E., Harrington W., Olson E., Reichard E. G. 2002. US Drinking Water Challenges in the Twenty-First Century. Environ Health Perspect, 110:43-52.

Liu W.T. and Lay C. 2007. Lab-on-a-chip devices for microbial monitoring and detection in water. Water science and technology: water supply, 7(2):165-172.

Maynard C. et al. 2005. Waterborne pathogen detection by use of oligonuclotide-based microarrays. Applied and Environmental Microbiology, 71(12):8548-8557.

McBean E. A. 2006. Failure mechanisms and monitoring methodologies pertinent for detection of exposure risks in water distribution networks. Security of Water Supply Systems: from Source to Tap. Springer.

Payment P. 1999. Poor efficacy of residual chlorine disinfectant in drinking water to inactivate waterborne pathogens in distribution systems. Canadian J of Microbiology, 45:709-715.

Pike W. 2004. Modeling drinking water quality violations with Baysian networks. J of the American water resource association, paper No.02085: 1563-1578.

Pilloti M., Menduni G., Castelli E. 1997. Monitoring the inception of sediment transport by image processing techniques. Experiments in Fluids, 23:202-208.

Schuster C. 2005. Infectious disease outbreaks related to drinking water in Canada, 19742001. Canadian J of public health, 96(4):254-258.

Shen Z. et al. 2007. Nonbalanced quartz crystal microbalance biosensor for bacterial detection using carbohydrate and lectin recognitions. Anal. Chem., 79:2312-2319.

Skansen J. et al. 2008. Distribution system on-line monitoring for detecting contamination and water quality change. J AWWA, 100(7):81-94.

Straub T. M., Chandler D. P. 2003. Towards a unified system for detecting waterborne pathogens. J Microbiol Methods, 53:185-197.

Ulitzer S., Lahav T., Ulitzur N. 2002. A novel and sensitive test for rapid determination of water toxicity. Environmental toxicology, 17(3):291-296. 
